\title{
Ischemic Stroke Lesion Segmentation by Analyzing MRI Images Using Deep Convolutional Neural Networks
}

\author{
${ }^{1}$ Shubham Joshi, ${ }^{2}$ Prof. Sonal Gore \\ ${ }^{1,2}$ Computer Department, Pimpri Chinchwad College of Engineering, Savitribai Phule Pune University, Pune \\ Email: spjoshi@live.com, sonalgore@gmail.com
}

Received: 09 ${ }^{\text {th }}$ July 2018, Accepted: $14^{\text {th }}$ August 2018, Published: $31^{\text {st }}$ August 2018

\begin{abstract}
Deep Learning proves to be the best way towards image classification tasks replacing ensembles with handcrafted features. It also has applications in medical imaging such as classification of tumorous and non-tumorous part from rest of the MRI image. Lesions is one such disease occurring in brain due to lack of oxygen during stroke. Automatic segmentation of ischemic stroke lesions is the perfect problem solved by using deep learning methods especially Convolution Neural Networks (CNNs). We present a patch based approach using multi-scale convolution layers with three pathways to segment lesions using 6 modalities of MRI images. The accuracy achieved using this method is comparable to other methods.
\end{abstract}

Keywords: Brain Tumor, Deep Learning, Segmentation, Convolution Neural Network, CNN

Introduction

Ischemic Stroke Lesions are caused by lack of oxygen to the brain during stroke and are major causes of disability and death globally. Eighty percent of strokes are caused due to cerebral ischemia. Various treatments currently available are surgery, chemotherapy, radiotherapy, or a combination of both of them. MRI is very useful in finding lesions in clinical practice, as we can acquire MRI sequences providing different information of brain tissues (S. Bauer et al., 2013). It is important to find the lesions in early stages so that good follow up treatment is given fast by doctors. However, segmenting these lesions (D. N. Louis et al., 2007) is time consuming task and requires highly qualified neurosurgeons. Thus, physicians usually use rough measures for evaluation. For these reasons, accurate semi-automatic or automatic methods are required (S. Bauer et al., 2013). However, it is a challenging task, since the shape, structure, and location of these abnormalities contain very high variability. Additionally, the tumor mass effect change the arrangement of the surrounding normal tissues (B. Menze et al., 2015). Also, MRI images present problems as intensity inhomogeneity, or different intensity ranges among the same sequences and acquisition scanners.
Various methods for brain tumor (lesions) segmentation tasks have been proposed and one method is using CNNs for this task as they have proven to best methods to handle such kind of tasks. These methods use labeled image data to extract features and use those for inference. Four different models for CNNs are compared in this review using various evaluation metrics such as dice score, sensitivity and specificity.

These methods are for multi-class pixel wise classification segmentation and we want to achieve a binary segmentation using and extending these methods. A novel patch wise three-pathway fully convolutional architecture is proposed for ischemic stroke lesion segmentation task.

Convolutional Neural Networks:

Convolution Neural Networks (CNNs) are a part of Deep Learning Methods which are good in extracting spatial features from an $\mathrm{n}$-dimensional array of basically any arbitrary size. CNNs are thought to be just a network of stacked layers, each layer performing some linear operation which should be differential so as to perform a so called training of that network on a dataset with huge samples (more samples in the database is always good!) which trains the hidden units (arbitrary neurons) in the network during training, to use those features to predict the given task on a new sample. Basically we learn the features for a given task and use those for the predictions on a similar new task. The research in CNNs have been focused at various hyper parameter optimizations and linear operations performed on the data including cascaded architectures.

The basic CNN architecture consists of Conv + Pool layers with activation functions and flattened fully connected layers connected together to a classifier. Using back propagation, we learn the features and then use those features for inference. A wide variety of research is being done in this field due to its promising nature in automation. Some of the research fronts in $\mathrm{CNN}$ have been discussed below.

Improvements in CNN's Architecture

Various variants of traditional CNNs have been proposed moving from simple layer stacking to complex cascaded CNNs. Some versions/ modifications of $\mathrm{CNN}$ are shown in Fig.1. As 
architecture progressed so did the layers as many different variants of convolution and pooling layers are also improved. Activation functions such as Adam and Adadelta are devised for fast learning moving from basic gradient descent. Also the fully connected layers can now be turned to fully convolution layers increasing the speed. Overfitting of networks was a major problem with deep and cascaded architectures but techniques like dropout helps in reducing over fitting by creating sparse connections. Various optimization techniques like Batch Normalization and Data Augmentation are used to optimize the network for various parameters like speed, rotational invariances and fast convergence of the network.

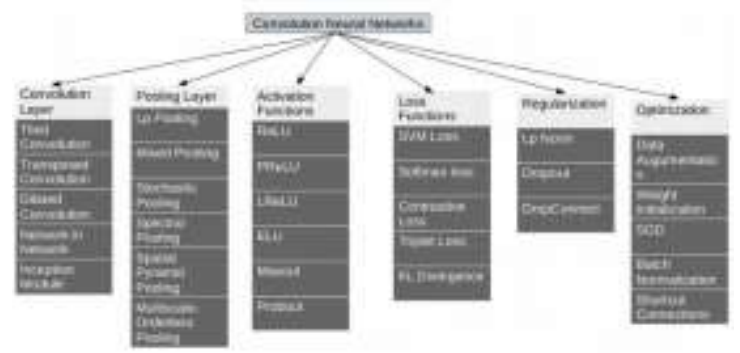

Fig. 1 Hierarchical Taxonomy of Modifications in Each Area in Convolution Neural Networks

\section{Applications of CNN in MRI Images}

Medical Imaging domain problems are very well suited to be solved using convolution neural networks. MRI imaging techniques (E. G. Van Meir et al., 2010) provides us with a good view of the internal structure for assessing various organs and anatomy structures of human body. Various tasks in medical MRI/CT imaging include various brain disease detection and segmentation, finding fractures in bones, various body cancer identification such as lung cancer, finding eye diseases etc. The availability of dataset is a major setback in medical imaging and very few dataset exists. Even if data exists, accurate labeled data is hard to be found and requires the manual work of medical persons.

Studies are being on various fronts in brain tumor and lung cancer detection. With the availability of datasets this will eventually be done on various fronts of medical imaging. CNN's have proved very good with image processing tasks but for medical imaging, we still need to work on them to achieve comparable accuracy to humans.

\section{Dataset and Evaluation Criteria:}

MICCAI BRaTS (B. Menze et al., 2015) organizes a competition for brain lesion segmentation task. The datasets available are ISLES 2015-2017. The dataset consists of 7 modalities of MRI data namely viz. DPWI, Tmax, TTP, CBV, CBF, MTT and ADC. Each brain sample consists each of these 7 modalities with their respective ground truth labels. Each modality of image and ground truth contains of 19 samples. Every ground truth label is annotated by expert neuro surgeons and contains annotations as lesion and nonlesion.

The evaluation of the segmented image is done by various metrics such as dice score, sensitivity, specificity and accuracy. The dice score, accuracy, sensitivity and specificity is given as shown in equation (1), (2), (3) and (4) respectively.

Dice $=2 T P / 2 T P+F P+F N$

Accuracy $=T P+T N / T P+F P+F N+T N$

Sensitivity $=T P / T P+F N$

Specificity $=T N / T N+F P$

where,

TP - true positive is pixels correctly segmented as foreground

FP - false positive is pixels falsely segmented as foreground

TN - true negative is pixels correctly detected as background

FN - false negative is pixels falsely detected as background

\section{Literature Survey:}

Various variants of traditional CNNs have emerged from the basic sequential CNNs to complex and cascaded CNNs. Cascaded CNNs perform various different convolution and pooling operations independently on input and merges those results for further layers. These architectures are seen to be working well. The basic architecture for brain tumor segmentation is given in Fig. 2.

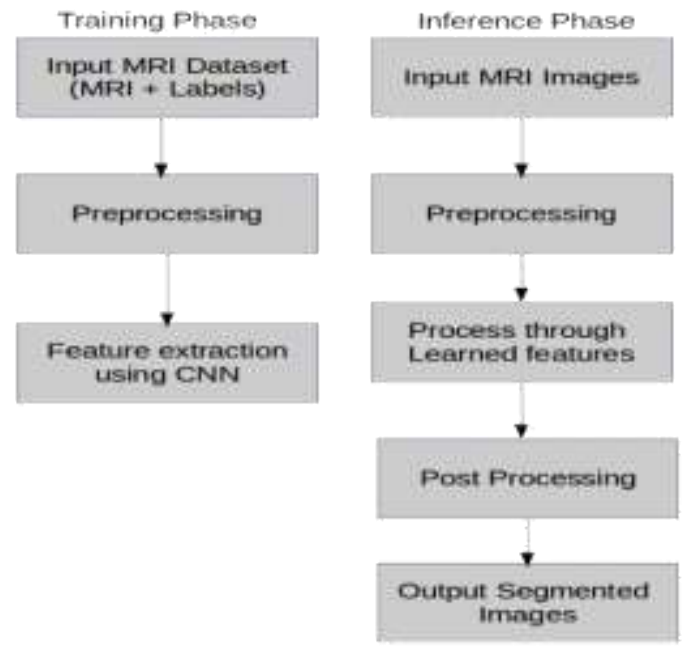

Fig. 2. Various Elements of Processing Pipeline in Training Phase (Left) and Inference Phase (Right) for Brain Tumor Segmentation

For the task of brain tumor segmentation (G. Tabatabai et al., 2010) various architectures using deep 
convolution neural networks have been proposed. (Sérgio Pereira et al., 2016) proposed a patch based method using $33 \times 33$ patches as input to $\mathrm{CNN}$. Two different architectures for HGG and LGG are proposed as LGG needs more care as it is prone to overfitting. The images were processed using N4ITK method (N. J. Tustison et al., 2010).Also intensity normalization is applied as given in L. G. Nyúl et al., 2000 and all the patches were made zero mean and unit variance. Data Augmentation was applied for rotational invariances. The architecture was simple but still achieved good results. The architectures consists of 3 Conv $(3 \times 3)$ layers with 64 filters followed by Max-pool (2x2) again followed by 3 Conv ( $3 \times 3)$ layers with 128 filters followed by Max-pool (2x2) connected by layers of fully connected network (128 hidden neurons each) followed 5 class softmax. Overfitting was reduced by using Dropout. LReLU activation was used for all convolution layers which performed well against ReLU. Using small kernel size with deeper architecture yielded good results.

Mohammad Havaei et al., 2016 used various cascaded CNN's architectures with different patch size. The input cascaded $\mathrm{CNN}$ of the two path version performed the best among all the cascaded CNNs proposed. A $65 \times 65$ patch with its centre $33 \times 33$ patch are taken as inputs. The dimensions of the $65 \times 65$ path is reduced to $5 \times 33 \times 33$ using a series of convolutions and poolings where we have 5 feature maps at. Then the reduced features are concatenated with $33 \times 33$ patches for further layers input. This enables us to capture both the local as well as global features of the brain MRI image thus getting better features. The concatenated $33 \times 33$ patches are then again given as input to two different CNNs. The number of parameters on this $\mathrm{CNN}$ is 802,368 . The architecture is fully convolutional and thus speeds up the training and inference process.

W. Mengqiao et al., 2017 devised a 3D CNN which is 22 layer deep. Average Pooling was used here which is less seen as we lose the actual information. The performance of this method was not above (Sérgio Pereira et al., 2016). This shows us increased layers don't help after a certain depth and cascaded architecture perform better. Also NT4IK preprocessing is necessary to obtain comparable accuracy using any model as every surveyed papers show their use.

S. Hussain et al., 2017 approached the problem in a similar way as (Mohammad Havaei et al., 2016) using patch size of $37 \times 37$ and centre 19x19 instead of $65 \times 65$ and $33 \times 33$. He might have thought of decreasing the train and inference time while using the patches of this size. Maxout was used instead of LReLU which increased the evaluation metrics but using convolutions of size $9 \times 9$ and $7 \times 7$ for $65 \times 65$ patches is not a good idea as it don't learns small representations instead convolutions of $5 \times 5$ and $3 \times 3$ should have been used. The performance of this model is still low compared to all above methods.

Inspired by these architectures a new architecture for lesion segmentation task is proposed in the next section.

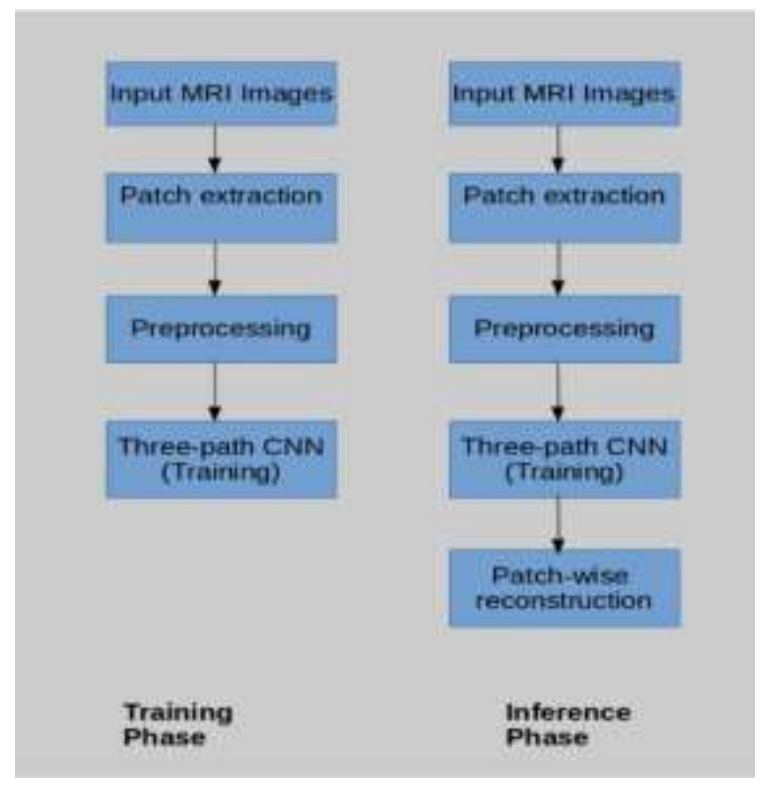

Fig. 3. Ischemic Stroke Lesion Segmentation
Architecture

\section{Proposed Method:}

Lesion segmentation task is a difficult task in biomedical imaging as the dataset is sparse i.e. there are more non-lesion part in labels than the lesion counterpart. For this reason we choose to work with patch based approach for given task. The proposed methodology used follows the architecture given in Fig.3.

We ignore the DPWI MRI image due to its size and constraints on memory. 33x33 patches are extracted from 6 modalities from every image. Thus, the input to our model will be of size $(6,33$, and 33$)$ where 6 refers to images of six modalities. Each image can contain 256x256 number of patches and thus a good sampling method is required.

First we tried out random sampling but the class imbalance was seen quickly and the model just learned to output the class with higher samples. Thus we choose balanced sampling to extract patches. Every patch was preprocessed to be zero mean and unit variance. The model architecture is shown in Fig.4.

The multiple paths help acquire the local as well as global features in patches and thus help the net to learn more effectively. This type of architecture is good for medical datasets having sparse labels. The number of parameters for our model is $14,812,738$. 
Helix Vol. 8(5): 3721- 3725

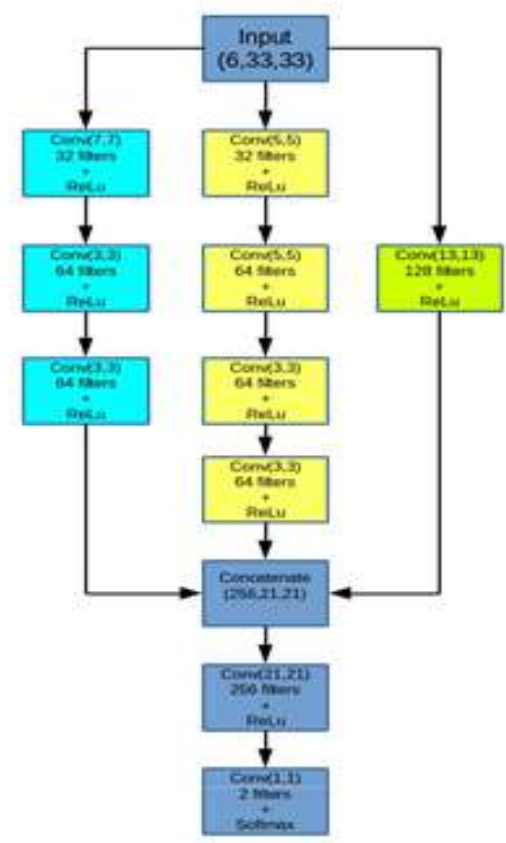

Fig. 4. Three Path CNN

Pre-Training

Training was done using 50000 patches. The data was equally balanced as the dataset is highly imbalanced. Adam optimizer with learning rate of 0.001 with decay of 0.005 is used and the network is trained using Nvidia 940MX GPU on batches of size 32. We trained for about 10 epochs as then the model started to overfit, each epoch took about 30 minutes. The model achieves $0.64 \%$ validation accuracy and the model starts to overfit.
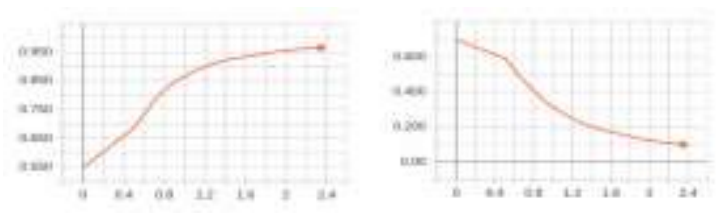

(a) Training Accuracy and Loss

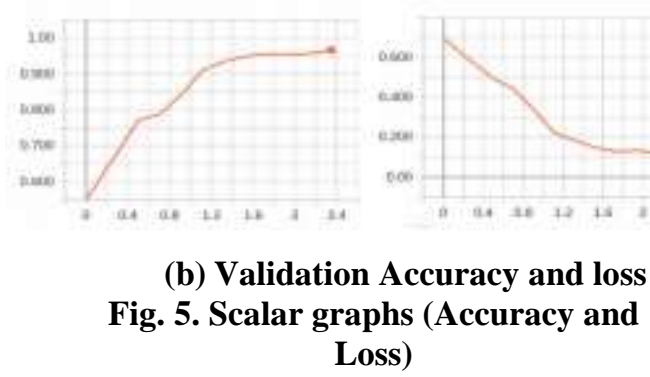

\begin{tabular}{|l|c|c|c|c|c|c|c|c|c|}
\hline \multicolumn{1}{|c|}{ Method } & \multicolumn{3}{|c|}{ Dice } & \multicolumn{3}{c|}{ Specificity } & \multicolumn{3}{c|}{ Sensitivity } \\
\hline & Complete & Core & Enhancing & Complete & Core & Enhancing & Complete & Core & Enhancing \\
\hline Sérgio Pereiraet al. & 0.88 & 0.83 & 0.77 & 0.88 & 0.87 & 0.74 & 0.89 & 0.83 & 0.81 \\
\hline Mohammad Havaei et al. & 0.85 & 0.78 & 0.73 & 0.93 & 0.80 & 0.72 & 0.80 & 0.76 & 0.75 \\
\hline W. Mengqiao et al. & 0.84 & 0.79 & 0.75 & 0.88 & 0.86 & 0.7 & 0.82 & 0.75 & 0.86 \\
\hline S.Hussain et al. & 0.80 & 0.67 & 0.85 & 0.85 & 0.82 & 0.88 & 0.82 & 0.63 & 0.83 \\
\hline This Work & 0.81 & 0.66 & 0.86 & 0.84 & 0.80 & 0.87 & 0.80 & 0.69 & 0.78 \\
\hline
\end{tabular}

Table 1: Results

\section{Fine-Tuning}

The model trained during pre-training uses balanced sampling and differs from the true distribution of our data, so we fine-tune our last convolution layer on the data sampled from the actual distribution of data i.e.( $0.12 \%$ of lesion part and the rest is non-lesion). The fine-tuning is done only for 10 epochs and then stopped due to overfitting. The optimizer used is SGD during fine tuning as it is seen that Adam does not converge after a certain point. The learning rate was kept low to 0.0001 , decay to 0.00001 and momentum to 0.97 . The model achieves $96 \%$ binary accuracy. The average dice score achieved on final predictions is 0.81 . The comparison of results to previous studies is shown in Table 1.

\section{Conclusion}

CNNs have drastically improved the task of image recognition, segmentation and tracking in image processing. The applications of these methods are helpful in variety of fields like object tracking, security, medical imaging etc. Cascaded CNNs especially have been helpful in medical imaging as these methods provide fast training and inference. Patches based method are good at imbalanced class segmentation problems but pose a lot of problems and requires a lot of fine tuning. Using patches increases the inference time by a very high margin. Thus the task of automatic lesion segmentation at practical level is still pending.

\section{References}

1. S. Bauer et al., "A survey of MRI-based medical image analysis for brain tumor studies," Phys. Med. Biol., vol. 58, no. 13, pp. 97-129, 2013.

2. D. N. Louis et al., "The 2007 who classification of tumours of the central nervous system," Acta Neuropathologica, vol. 114, no. 2, pp. 97-109, 2007 
Helix Vol. 8(5): 3721- 3725

3. E. G. Van Meir et al., "Exciting new advances in neuro-oncology: The avenue to a cure for malignant glioma," CA, Cancer J. Clinicians, vol. 60, no. 3, pp. 166-193, 2010.

4. G. Tabatabai et al., "Molecular diagnostics of gliomas: The clinical perspective," Acta Neuropathologica, vol. 120, no. 5, pp. 585-592, 2010. 5. B. Menze et al., "The multimodal brain tumor image segmentation benchmark (BRATS)", IEEE Trans. Med. Imag., vol. 34, no. 10, pp. 1993-2024, Oct. 2015.

6. Sérgio Pereira et al. "Brain Tumor Segmentation Using Convolutional Neural Networks in MRI Images", IEEE Trans. Med. Imag., vol. 35, no. 5, pp. 0278-0062, May. 2016 